\title{
Optimal absorber thickness in long-wave multiple-stage detector
}

\author{
Klaudia Hackiewicz $^{1}$ (D) P Piotr Martyniuk ${ }^{1}$. Jarosław Rutkowski ${ }^{1}$
}

Received: 6 April 2018 / Accepted: 4 February 2019 / Published online: 10 February 2019

(c) The Author(s) 2019

\begin{abstract}
The detectivity characteristics of interband cascade infrared type-II superlattice detectors for long-wave infrared detection $\left(\lambda_{\text {cut-off }}=8 \mu \mathrm{m}\right.$ at room temperature) are discussed. We present comparison of two superlattices: InAs/GaSb and InAs/InAsSb, assuming the characteristic parameters-absorption coefficients $\alpha$ and carrier lifetimes $\tau$ published in literature. Dependence of the Johnson-noise limited detectivity on the absorber thickness for a different number of stages is reported. Higher detectivity $D^{*}$ value can be achieved by increasing the carrier lifetime. However, for detectors based on type-II InAs/GaSb superlattice increasing the carrier lifetime up to $25 \mathrm{~ns}$ leads to a situation in which one stage is preferred, i.e. for detector with a single absorber, we obtain the highest detectivity value. In the case of InAs/InAsSb material, the situation is similar for $\tau \geq 80 \mathrm{~ns}$. We have shown that the optimal absorber thickness at which the highest detectivity values are obtained depends not only on the absorption coefficient $\alpha$ and the number of stages $N_{S}$, but also on the carrier diffusion length $L$. According to a calculations, cascade detectors based on Ga-free material should have much higher optimal absorber thicknesses than materials based on InAs/ $\mathrm{GaSb}$.
\end{abstract}

Keywords Interband cascade infrared detectors · IB CID · T2SL InAs/GaSb · T2SL InAs/ InAsSb $\cdot$ LWIR detection

\section{Introduction}

The search for novel infrared detector materials, as well as new detector architectures, is motivated by the need to increase background limited operating temperatures, thereby lowering impact of bulky and expensive coolers. The commonly used and known material for infrared detectors, which is $\mathrm{HgCdTe}$ in contrast to the materials from group III-V requires a reduced operating temperature. Type-II superalttices (T2SLs) InAs/GaSb (Rogalski et al. 2017; Ye et al. 2015; Lei et al. 2016; Huang et al. 2017) have recently gained a lot of interest and are considered as a viable alternative $\mathrm{HgCdTe}$ technology in the long-wave infrared

Klaudia Hackiewicz

klaudia.hackiewicz@wat.edu.pl

1 Institute of Applied Physics, Military University of Technology, 2 Urbanowicza Str., 00908 Warsaw, Poland 
(LWIR) detection. The advantages of the type-II superlattices (T2SLs) include the suppressions of Auger recombination (Ashley and Elliott 1985) and tunneling current (Nguyen et al. 2007; Bogdanov et al. 2011), and high uniformity (Nguyen et al. 2011). Increasing minority carrier lifetimes relative to bulk $\mathrm{HgCdTe}$ will result in increased detectivities at the same operating temperature, or the same detectivity at higher operating temperature. Short minority carrier lifetime in T2SLs InAs/GaSb has recently been partially attributed to acceptor like defects in GaSb rather than the interfaces. With Ga being the suspected origin of the short minority carrier lifetime (Steenbergen et al. 2012), the "Ga-free" T2SLs InAs/InAsSb have been proposed as another alternative material that has the potential for longer lifetimes (Rogalski et al. 2017; Belenky et al. 2011; Grein et al. 1995; Pellegrino and DeWames 2009; Connelly et al. 2010; Donetsky et al. 2010; Steenbergen et al. 2011).

Nevertheless, T2SLs have their own important shortcomings affecting the performance of the detector, which is limited by diffusion length. This issue can be overcome utilizing a multiple-stage photodetector with multiple short absorbers-interband (IB) cascade infrared detectors (CIDs). IB CIDs contain multiple-discrete absorber connected in series using interband tunneling heterostructure. Each absorber can be shorter than the diffusion length, so the incident photons can be effectively collected. The thin discrete absorbers and series connections make the device resistance high so that noise is suppressed and the photocurrent flows more easily to an external circuit, resulting in the ability to high-temperature and high-speed operation (Hinkey and Yang 2013; Yang et al. 2010).

In this work, we report on the dependence of the Johnson-noise limited detectivity on the absorber thickness for a different number of stages, assuming performance of LWIR T2SLs, which can be useful to find the best detector architecture to reach high value of detectivity. We apply well known general theory for photovoltaic cells to multiple-stage interband detectors and obtain the aforementioned dependences.

\section{Modeling}

Infrared photodetectors operating under various circumstances are characterized by the detectivity $D^{*}$ which is the normalized signal-to-noise ratio performance. The detectivity of photodetectors operated at a low background and that of near room temperature operation is limited by the noise arising from the statistical nature of thermal generation-recombination processes in semiconductors. The value of $D^{*}$ is proportional to the inverse of the noise-equivalent power and is normalized for a detector of a given area $A$ and bandwidth $\Delta f$, and can be given by:

$$
D^{*}=\frac{q \lambda}{h c} \eta_{e} \sqrt{\frac{A \Delta f}{i_{n}^{2}}},
$$

where $q, \lambda, h, c, \eta_{e}$ and $i_{n}$ are elementary charge, wavelength, Planck constant, speed of light, detector's external quantum efficiency and noise current, respectively. As can be seen from above formula lot of parameters influence on the value of the thermal noiselimited $D^{*}$, but the most important are absorber thickness $d$, absorption coefficient $\alpha$ and minority carrier diffusion length $L$ contributing to $\eta_{e}$. All these parameters strongly depend on the type of material that is selected. There is a strong coupling between the thickness of the absorber and the number of stages, what is shown in this paper. In this work, we determine the optimal number of stages $N_{s}$ depending on the individual absorber thickness 
$d$, assuming identical absorbers in each stage, in order to obtain maximum detectivity in LWIR range. The detectivity $D^{*}$ for that kind of photodetector, assuming zero surface recombination velocity and that absorber is illuminated from the collection point, is given by (Donetsky et al. 2010):

$$
D^{*}\left(N_{s}, d\right)=\frac{\lambda}{h c} \sqrt{N_{S}} \frac{\alpha L}{1-(\alpha L)^{2}} \frac{\left[\sinh (d / L)+\alpha L e^{-\alpha d}-\alpha L \cosh (d / L)\right] / \cosh (d / L)}{\sqrt{4 g_{t h} L \tanh (d / L)}} e^{-\alpha d\left(N_{S}-1\right)}
$$

where $g_{t h}$ is thermal carrier generation rate per unit volume. Based on the above equation the number of stages $N_{s}$ at which $D^{*}$ reaches its maximum, related with the optimal absorber thickness $d_{\text {opt }}$ can be determined by the formula:

$$
d_{\text {opt }}=\frac{1}{2 N_{s} \alpha} \text {. }
$$

Like was mentioned before, beyond just the capability of tuning the optical bandgap, the superlattice structure provides the added capability of energy band structure engineering. This capability to shape the fundamental physics of the material is not available in compositional alloys like $\mathrm{HgCdTe}$. By examining the band structure of these equivalent designs the optimum design can be selected, according to the equations, to enhance important parameters such as the absorption coefficient $\alpha$, carrier lifetime $\tau$, and therefore the associated diffusion length $L$ or electron/hole effective mass. All these important parameters affecting the shape and maximum value of $D^{*}$, depending on the author, are very different. According to Vurgaftman et al. (2016) at $\lambda=8 \mu \mathrm{m}$ the absorption coefficient for T2SL InAs/GaSb and InAs/InAsSb is 1250 and $700 \mathrm{~cm}^{-1}$, respectively. However, the addition of indium to the GaSb layers can increase the absorption coefficient of the superlattice material, what was shown by Brown et al. (2004) Currently, in T2SLs the minority carrier lifetime at $77 \mathrm{~K}$ for LWIR range is typically between 10 and $40 \mathrm{~ns}$ for InAs/GaSb and order of magnitude higher lifetimes in InAs/InAsSb (Belenky et al. 2011; Grein et al. 1995; Pellegrino and DeWames 2009; Connelly et al. 2010; Donetsky et al. 2010; Steenbergen et al. 2011). Thus, in ambient temperature carrier lifetimes are much lower. We assumed $\tau=3 \mathrm{~ns}$ for InAs/GaSb and $\tau=30 \mathrm{~ns}$ for InAs/InAsSb at $300 \mathrm{~K}$. Figure 1 shows comparison of detectivity for two T2SLs: InAs/GaSb and InAs/InAsSb versus absorber thickness for different number of stages $N_{S}$ at room temperature.

(a)

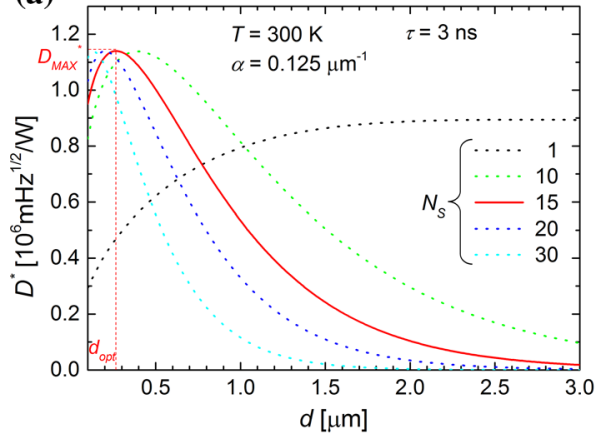

(b)

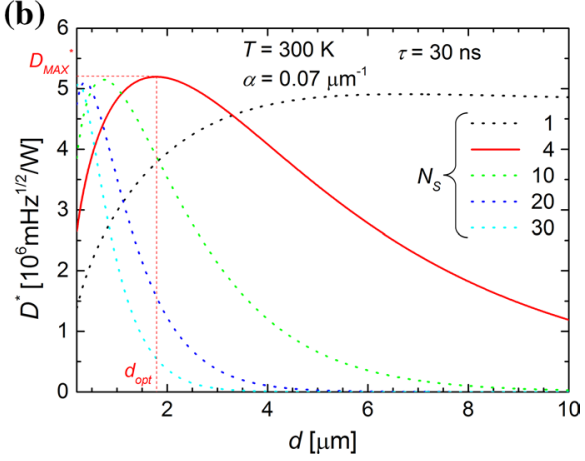

Fig. 1 Detectivity versus absorber thickness for T2SL a InAs/GaSb and b InAs/InAsSb at $300 \mathrm{~K}$ 
(a)

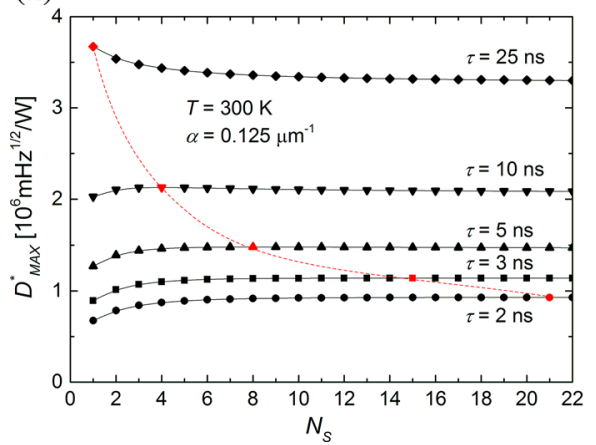

(b)

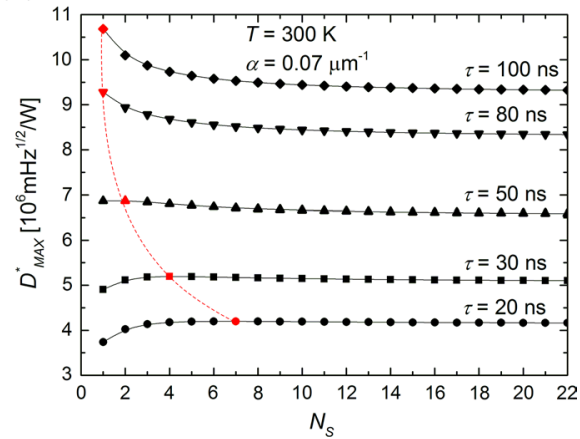

Fig. $2 D_{M A X}^{*}$ versus number of stages $N_{S}$ for T2SL a InAs/GaSb and $\mathbf{b}$ InAs/InAsSb. (Color figure online)

(a)

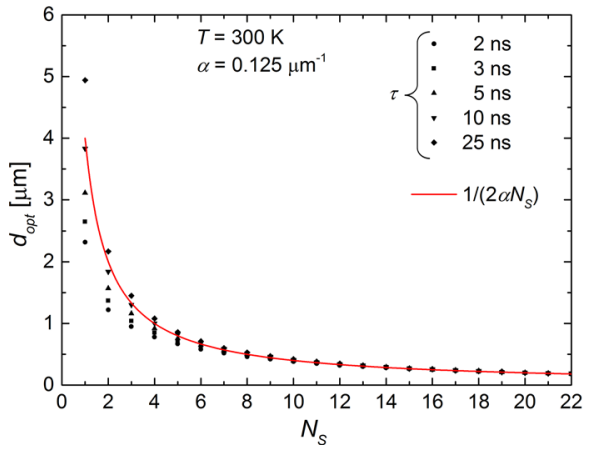

(b)

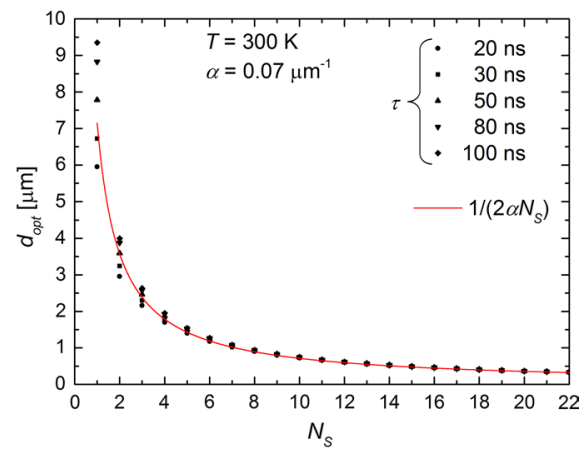

Fig. 3 Optimal absorber thickness $d_{\text {opt }}$ versus $N_{S}$ for T2SL a InAs/GaSb and b InAs/InAsSb. (Color figure online)

According to the Fig. 1a, in the case of T2SL InAs/GaSb $D^{*}$ reaches the highest value for 15 stages, while for T2SL InAs/InAsSb it is only for 4 stages (Fig. 1b). As can be seen, the maximum value of detectivity does not change significantly with number of stages. As the number of cascades increases, the optimal thickness of the absorber $d_{\text {opt }}$ at which detectivity reaches the maximum decreases. Optimal absorber thickness at which $D^{*}$ reaches the highest possible value in investigated range is much higher for $\mathrm{Ga}$-free material than for a T2SL InAs/GaSb and is equal 1.78 and $0.27 \mu \mathrm{m}$, respectively. Regardless of the number of cascades, detectors from the InAs/InAsSb superlattice reach five times better detectivity values. Due to the fact that the carrier lifetime is not well known, and many authors give significantly different values, Fig. 2 assumes improvement or worsening of lifetimes in both cases. As can be seen from the Fig. 2, as the carrier lifetime raises, detectivity is increased. For detectors based on T2SL InAs/GaSb the optimal number of stages is 21, 15, 8 and 4 for the carrier lifetimes 2, 3, 5 and $10 \mathrm{~ns}$, respectively. However, increasing the carrier lifetime up to $25 \mathrm{~ns}$ leads to a situation in which one stage is preferred, i.e. for detector with a single absorber, we obtain the highest detectivity value. In the case of InAs/InAsSb material, the value of $\tau \geq 80 \mathrm{~ns}$ causes such a situation [see red markers and dashed line in Fig. 2b]. 
The maximum values of detectivity $D_{M}^{*}$ ffrom Fig. $2 \mathrm{a}$, b correspond to some optimal values of absorber thickness, as shown in Fig. 3a, b, respectively. In the Fig. 3, the red curves of the optimal absorber thickness for a given number of stages resulting from Eq. (3) are marked. The presented data shows that this formula is not valid for small $N_{S}$ values, because the optimal absorber thickness also depends on the carrier lifetime. The optimal absorber thickness increases with increasing carrier lifetime. The changes in optimal absorber thickness are significant for small $N_{S}$. For the number of stage exceeding 10, Eq. (3) is fulfilled. Equation (3) results from the determination of the maximum of function $D^{*}\left(N_{S} d\right)$ [Eq. 2] in relation to $N_{S}$, assuming constancy $d$. However, for small number of stages, changes in thickness are large and therefore the thickness $d_{o p t}$ depends not only on $N_{S}$ and $\alpha$, but also on diffusion length $L$, and thus carrier lifetime $\tau$. This dependence is stronger in the case of a material with shorter carrier lifetime, i.e. T2SL InAs/GaSb [see Fig. 3] and higher operating temperatures of the detector.

\section{Conclusions}

In this work, we reported on the dependence of the Johnson-noise limited detectivity on the absorber thickness for a different number of stages, assuming performance of LWIR T2SLs. We have shown that the optimal absorber thickness at which the highest detectivity values are obtained depends not only on the absorption coefficient $\alpha$ and the number of stages $N_{S}$, but also on the carrier diffusion length $L$. A comparison of the two types of the superlattice showed that the detector based on InAs/InAsSb reaches higher detectivity than the detectors based on InAs/GaSb, even for fewer cascades. High temperatures and lower carrier lifetimes lead to the diffusion length shortening. Thus, in such cases, multiple-stage approach should be beneficial. Nevertheless, even if the detectivity is greater for a single absorber detector, the cascade detectors are characterized by a higher speed of operation. Thus, the normalized signal-to-noise ratio is only one of the parameters that can be optimized. In order to improve the speed of the detector's operation, its architecture should also be optimized, including the thickness of individual absorbers.

Acknowledgements We acknowledge support by The National Science Centre-the Grant No. OPUS/ UMO-2015/19/B/ST7/02200.

Open Access This article is distributed under the terms of the Creative Commons Attribution 4.0 International License (http://creativecommons.org/licenses/by/4.0/), which permits unrestricted use, distribution, and reproduction in any medium, provided you give appropriate credit to the original author(s) and the source, provide a link to the Creative Commons license, and indicate if changes were made.

\section{References}

Ashley, T., Elliott, C.T.: Nonequilibrium devices for infrared detection. Electron. Lett. 21, 451-452 (1985)

Belenky, G., Kipshidze, G., Donetsky, D., Svensson, S.P., Sarney, W.L.: Effects of carrier concentration and phonon energy on carrier lifetime in type-2 SLS and properties of $\operatorname{InAs}_{1-\mathrm{x}} \mathrm{Sb}_{\mathrm{x}}$ alloys. Proc. SPIE 8012, 80120W (2011)

Bogdanov, S., Nguyen, B.-M., Hoang, A.M., Razeghi, M.: Surface leakage current reduction in long wavelength infrared type-II InAs/GaSb superlattice photodiodes. Appl. Phys. Lett. 98, 183501 (2011) 
Brown, G.J., Houston, S., Szmulowicz, F.: Type-II InAs/GaSb superlattices for very long wavelength infrared detectors. Phys. E 20, 471-474 (2004)

Connelly, B.C., Metcalfe, G.D., Shen, H., Wraback, M.: Direct minority carrier lifetime measurements and recombination mechanisms in long-wave infrared type II superlattices using time-resolved photoluminescence. Appl. Phys. Lett. 97, 251117 (2010)

Donetsky, D., Belenky, G., Svensson, S., Suchalkin, S.: Minority carrier lifetime in type-2 InAs-GaSb strained-layer superlattices and bulk HgCdTe materials. Appl. Phys. Lett. 97, 052108 (2010)

Grein, C.H., Young, P.M., Flatté, M.E., Ehrenreich, H.: Long wavelength InAs/InAsSb infrared detectors: optimization of carrier lifetimes. J. Appl. Phys. 78(12), 7143-7152 (1995)

Hinkey, R.T., Yang, R.Q.: Theory of multiple-stage interband photovoltaic devices and ultimate performance limit comparison of multiple-stage and single-stage interband infrared detectors. J. Appl. Phys. 114(10), 104506 (2013)

Huang, W., Lei, L., Li, L., Massengale, J.A., Yang, R.Q., Mishima, T.D., Santos, M.B.: Current-matching versus non-current-matching in long wavelength interband cascade infrared photodetectors. J. Appl. Phys. 122, 083102 (2017)

Lei, L., Li, L., Ye, H., Lofti, H., Yang, R.Q., Johnson, M.B., Massengale, J.A., Mishima, T.D., Santos, M.B.: Long wavelength interband cascade infrared photodetectors operating at high temperatures. J. Appl. Phys. 120, 193102 (2016)

Nguyen, B.-M., Hoffman, D., Delaunay, P.-Y., Razeghi, M.: Dark current suppression in type II InAs/GaSb superlattice long wavelength infrared photodiodes with M-structure barrier. Appl. Phys. Lett. 91(16), $163511(2007)$

Nguyen, B.-M., Chen, G., Hoang, M.-A., Razeghi, M.: Growth and characterization of long-wavelength infrared type-II superlattice photodiodes on a 3-in GaSb wafer. IEEE J. Quantum Electron. 47(5), 686690 (2011)

Pellegrino, J., DeWames, R.: Minority carrier lifetime characteristic in type II InAs/GaSb LWIR superlattice $\mathrm{n}+\pi \mathrm{p}+$ photodiodes. Proc. SPIE 7298, 72981U (2009)

Rogalski, A., Kopytko, M., Martyniuk, P.: InAs/GaSb type-II superalttice infrared detectors: three decades of development. Proc. SPIE 10177, 1017715 (2017)

Steenbergen, E.H., Connelly, B.C., Metcalfe, G.D., Shen, H., Wraback, M., Lubyshev, D., Qiu, Y., Fastenau, J.M., Liu, A.W.K., Elhamri, S., Cellek, O.O., Zhang, Y.-H.: Significantly improved minority carrier lifetime observed in a long-wavelength infrared III-V type-II superlattice comprised of InAs/InAsSb. Appl. Phys. Lett. 99(25), 251110 (2011)

Steenbergen, E.H., Nunna, K., Ouyang, L., Ullrich, B., Huffaker, D.L., Smith, D.J., Zhang, Y.-H.: Strainbalanced InAs/InAs ${ }_{1-x} \mathrm{Sb}_{\mathrm{x}}$ type-II superlattices grown by molecular beam epitaxy on GaSb substrates. J. Vac. Sci. Technol. B 30(2), 02B107 (2012)

Vurgaftman, I., Belenky, G., Lin, Y., Donetsky, D., Shterengas, L., Kipshidze, G., Sarney, W.L., Svensson, S.P.: Interband absorption strength in long-wave infrared type-II superlattices with small and large superlattice periods compared to bulk materials. Appl. Phys. Lett. 108, 222101 (2016)

Yang, R.Q., Tian, Z., Cai, Z., Klem, J.F., Johnson, M.B., Liu, H.C.: Interband-cascade infrared photodetectors with superlattice absorbers. J. Appl. Phys. 107, 054514 (2010)

Ye, H., Li, L., Lofti, H., Lei, L., Yang, R.Q., Keay, J.C., Mishima, T.D., Santos, M.B., Johnson, M.B.: Molecular beam epitaxy of interband cascade structures with InAs/GaSb superlattice absorbers for long-wavelength infrared detection. Semicond. Sci. Technol. 30, 105029 (2015)

Publisher's Note Springer Nature remains neutral with regard to jurisdictional claims in published maps and institutional affiliations. 